\title{
Numerical investigation of the flow over a model transonic turbine blade tip
}

\author{
By A NDREW P. S. WHEELER ${ }^{1} \dagger$ \\ RICHARD D. SANDBERG ${ }^{2}$ \\ ${ }^{1}$ Department of Engineering, University of Cambridge, \\ Trumpington Street, Cambridge, U.K. \\ ${ }^{2}$ Department of Mechanical Engineering, University of Melbourne, Victoria 3010, Australia
}

(Received ?? and in revised form ??)

Direct numerical simulations are used to investigate the unsteady flow over a model turbine blade tip at engine scale Reynolds and Mach numbers. The DNS are performed with an in-house multi-block structured compressible Navier-Stokes solver. The particular case of a transonic tip flow is studied since previous work has suggested compressibility has an important effect on the turbulent nature of the separation bubble at the inlet to the tip-casing gap and subsequent flow reattachment. The flow is simulated over an idealized tip geometry where the tip gap is represented by a constant area channel with a sharp inlet corner to represent the pressure side edge of the turbine blade. The effects of free-stream disturbances, cross-flow and pressure-side boundary-layer on the tip flow aerodynamics and heat transfer are studied. For 'clean' in-flow cases we find that even at engine scale Reynolds numbers the tip flow is intermittent in nature, i.e. neither laminar nor fully turbulent. The breakdown to turbulence occurs through the development of spanwise streaks with wavelengths around $15-20 \%$ of the gap height. Multi-dimensional linear stability analysis confirms the two-dimensional base state to be most unstable with respect to spanwise wavelengths of $25 \%$ of the gap height. The linear stability analysis also shows that addition of cross-flows with $25 \%$ of the streamwise gap exit velocity increase the stability of the tip flow. This is confirmed by the DNS which also show that the turbulence production is significantly reduced in the separation bubble. For the case when free-stream disturbances are added to the inlet flow, viscous dissipation and the rapid acceleration of the flow at the inlet to the tip-casing gap causes significant distortion of the vorticity field and reductions of turbulence intensity as the flow enters the tip gap. The DNS results also suggest that the assumption of the Reynolds analogy and a constant recovery factor are not accurate, in particular in regions where the skin friction approaches zero while significant temperature gradients remain, such as in the vicinity of flow reattachment.

\section{Introduction}

Here we present direct numerical simulations (DNS) of a transonic turbine tip flow with free-stream disturbances at engine representative Mach and Reynolds numbers in order to determine the effects of turbulence on the tip heat transfer and aerodynamics. Turbine blade tips can experience significant degradation throughout the engine life-cycle due to the high mechanical and thermal stresses in the tip region. The high thermal loads are largely due to the tip-leakage flow, which forms in the gap between the moving rotor

$\dagger$ Email address for correspondence: aw329@cam.ac.uk 


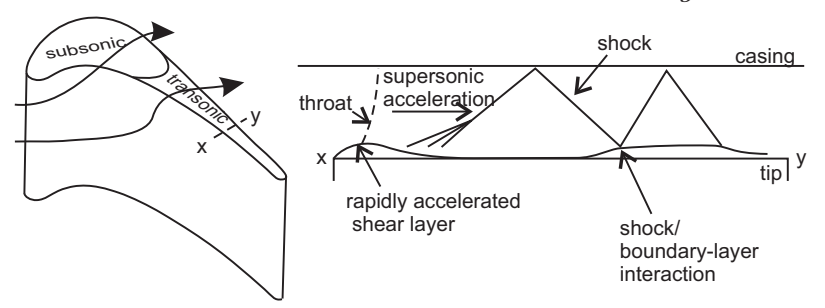

FIgURE 1. Schematic of a transonic tip flow

blade tips and the casing of the machine. Turbine tip flows are also responsible for up to $30 \%$ of stage losses for newly installed engines (Harvey (2004)). In-service, the blade tips are eroded due to chemical attack, fatigue and creep which are all exacerbated by the high gas temperatures, and this causes further performance degradation (Bunker (2004)). Predicting the correct heat transfer to the tip is consequently a critical part of the turbine design process. Shroudless high pressure (HP) turbine blades can have peak Mach numbers within the tip gap of 1.8 and, thus, supersonic effects cannot generally be ignored (Wheeler et al. 2011; Zhang et al. 2011; Moore et al. 1989; Moore \& Elward 1993). Supersonic effects tend to occur over the aft region of the tip where the blade loading is highest, although the extent of supersonic flow over the tip is strongly dependent on the tip geometry (Coull et al. 2014; Zhang et al. 2014). This area of the tip is important because the blade thickness in this region is small which means complex tip geometries are often not feasible and this zone is typically difficult to cool. The basic tip flow structure in this region is shown in Figure 1. As the flow enters the tip gap, the flow separates from the pressure side corner. The separation accelerates the flow to supersonic conditions like a converging-diverging nozzle, thus as the separation begins to reattach the change in area forces the mainstream flow to accelerate. This in-turn aids the reattachment (Wheeler et al. 2011). Compression waves form due to the curvature of the reattaching boundary-layer. These coalesce into an oblique shock which reflects from the tip and casing, leading to shock/boundary-layer interactions which can cause further boundary-layer separation. The reattachment process of the separation bubble at the inlet to the gap is important because it will influence the heat transfer over the remainder of the tip. From extensive data on subsonic tip flows it is known that high levels of turbulent mixing in the reattachment zone lead to high heat transfer levels downstream of the separation bubble (Bunker 2004). However, the extent to which heat transfer levels are diminished when the separation bubble reattachment occurs through supersonic acceleration has not yet been reported in detail, although the results of (Zhang \& He 2013; De Maesschalck et al. 2015, 2014) do suggest that this supersonic acceleration can be exploited to reduce tip heat load. Wheeler \& Sandberg (2012) recently presented DNS results showing the intermittent nature of the transonic tip flow, fluctuating between laminar and turbulent flow downstream of the bubble reattachment.

The effects of turbulence on tip heat transfer are likely to be important, but it is interesting to note that although Mach numbers are high in the tip gap, the Reynolds number of the tip flow will be generally low (based on gap height, $g, R e_{g} \sim 2 \times 10^{4}$ ). The low Reynolds number and the presence of strong accelerating pressure gradients downstream of the separation raises the possibility that the transonic tip flow may not be fully turbulent. This is important because the ability of turbulence models to cope with transitional flows is questionable, and so this raises questions about the use of RANS methods for the prediction of blade tip heat transfer.

This paper aims to use direct numerical simulation to solve the turbulent unsteadiness 
and therefore fully resolve the boundary-layer flow on the tip. The objective is to determine the sensitivity of the tip flow to incoming disturbances. In order to more accurately represent the actual flow configuration of a high pressure turbine tip gap, cross-flow and a pressure surface boundary layer were also included in the simulations. The flow is simulated over an idealized tip geometry where the tip gap is represented by a constant area channel with a sharp inlet corner to represent the pressure side edge of the turbine blade. The paper is structured as follows. The compressible code used in this study is introduced in $\S 2$. The set-up of a engine representative tip flow and grid sensitivity are described in $\S 3$. In $\S 4$, DNS results, linear stability analysis and Reynolds number dependence are presented for the datum case. This is followed by an investigation of the effects of free-stream disturbances, cross-flow and pressure-surface boundary-layer on the tip heat transfer and skin friction in $\S 5$. The paper concludes with a discussion of the most important results in $\S 6$.

\section{Solution algorithm and data processing}

The DNS were performed with an in-house multi-block structured compressible NavierStokes solver purposely developed for exploiting high performance computing systems. The numerical method comprises a five step, fourth order accurate low storage RungeKutta method (Kennedy et al. 2000), with a Fourier method for discretization of the spanwise direction. Spatial derivatives in the streamwise and lateral directions are discretised using a central fourth-order accurate finite difference scheme with energy conserving boundary schemes of the same formal order (Carpenter et al. 1999). Additionally, a skew-symmetric splitting (Kennedy \& Gruber 2008) is used to stabilize the convective terms. The code has been validated against a number of benchmark cases and used, among other applications, for DNS of supersonic axisymmetric wakes (Sandberg 2012) and turbulent jets in coflow (Sandberg et al. 2012). More recently, the code has also been applied to low pressure turbine flows (Sandberg et al. 2015), transonic tip flows (Wheeler \& Sandberg 2012) and high pressure transonic turbine flows (Wheeler et al. 2016).

Surface heat flux is presented as a normalized heat flux defined in equation 2.1:

$$
q_{\text {norm }}=\frac{q g}{\left(T_{o}-T_{w}\right) k_{r e f}},
$$

where $q$ is the surface heat flux, $T_{o}$ is the freestream stagnation temperature, $T_{w}$ is the wall temperature and $k_{r e f}$ is a reference conductivity, taken to be at the gap-exit static temperature in the freestream. Some results presented are shown in terms of isentropic Mach number, which is the Mach number determined from the local surface static pressure and inlet total pressure. The heat transfer coefficient $(h)$ is determined by performing simulations at two wall temperatures $\left(T_{w} / T_{o}=1.03\right.$ and 0.89$)$ and calculating the difference in local heat transfer at the two different wall temperatures (i.e., $h=\frac{\Delta q}{\Delta T_{w}}$ ). The heat transfer coefficient is presented in the paper as a Nusselt number, consistent with the definition of Reynolds number,

$$
N u=\frac{h g}{k_{r e f}},
$$

Results of skin friction are also shown and these are determined using the local timeaveraged wall shear stress and the local boundary-layer edge velocity and density. All quantities presented in this paper are non-dimensionalized based on reference conditions at the gap exit and a length scale equal to the gap height $(g)$. Velocities are therefore normalized by the free-stream velocity at gap exit $\left(u_{r e f}\right)$, turbulent kinetic energy is nor- 
malized by $u_{r e f}^{2}$, vorticity is normalized by $u_{r e f} / g$. Time is normalized by $g / u_{r e f}$ and thus one unit represents the time taken for the flow to pass through a length equal to the height of the gap. Likewise, frequency $f$ is given as a non-dimensional quantity, using reference velocity and gap-height to non-dimensionalize. Power spectral density of velocity magnitude, $P_{x x}$, is normalized by $u_{r e f}^{2}$. The Reynolds number is defined based on these reference conditions $R e_{g}=\rho_{\text {ref }} u_{r e f} g / \mu_{r e f}$. For the cases with cross-flow, the Reynolds number is based on the freestream velocity magnitude at gap exit $R e_{g}=\rho_{\text {ref }} \sqrt{u_{r e f}^{2}+w_{r e f}^{2}} g / \mu_{\text {ref }}$. Since, for the crossflow cases, the spanwise component of velocity was $w_{r e f}=0.25 u_{\text {ref }}$ the Reynolds number based on the $x$-component of velocity was $4 \%$ lower than the cases without crossflow.

Statistical quantities were determined using long time-series data to ensure convergence of the statistical quantities. By comparing the statistics obtained over a number of subsequent time intervals to each other it was found that statistical convergence was obtained using long time-series data over a nondimensional time of $\Delta t=50$. During the solutions, Favre-based time integration was used to determine the time-averaged flow field and turbulence quantities.

\section{Numerical set-up of an engine scale tip flow}

Direct numerical simulations were performed at Reynolds numbers of $1 \times 10^{4} \leqslant R e_{g} \leqslant$ $2 \times 10^{4}$, based on gap exit conditions and gap height. Typical HP blade Reynolds numbers based on blade-exit conditions are around $1-2 \times 10^{6}$ with gap heights in the range of $1 \%$ span. The tip-gap Reynolds number in this study is thus in the engine representative range for low aspect ratio blading; DNS predictions of the effect of tip-gap Reynolds number were discussed previously by the authors in Wheeler \& Sandberg (2012).

A schematic of the computational domain is shown in figure 2 . The flow is simulated over an idealized tip geometry. In this geometry, the tip gap is represented by a constant area channel with a sharp inlet corner to represent the pressure side edge of the turbine blade. This representation of a turbine tip flow has been used several times in previous work (for instance see Chen et al. (1993)). An example of a typical flow pattern is shown in figure 3 . The flow separates at the sharp inlet edge causing the characteristic separation bubble found in most tip gap flows. The formation of shock waves is typical of the tip gap flows found in transonic blade rows (for example Wheeler et al. 2011; Zhang et al. 2011; Moore \& Elward 1993). A tip width-to-gap ratio of 5 was chosen, which is similar to the tip geometry near the blade trailing edge. This region was chosen because here accurate prediction of the physical mechanisms of the flow and the heat transfer are particularly important for the above mentioned reasons. The upstream plenum dimensions in the $x$ and $y$ directions were chosen to be $5 g$ and $6 g$ respectively. The effect of plenum size was tested with steady RANS simulations using the FLUENT v16.0 density-based $2^{\text {nd }}$ order solver, with a Spalart-Allmaras turbulence model; increasing the plenum size from $5 \times 6 g$ to $10 \times 11 g$ was found to have minimal effects on the flow structures in the tip-gap, such as shock positions and separation bubble size.

Characteristic boundary-conditions were applied at the exit to avoid reflections from this boundary. A zonal characteristic boundary-condition (Sandberg \& Sandham 2006) was applied over the final 50 streamwise grid lines, i.e. to the last $0.25 \mathrm{~g}$ of the computational domain, which has been previously shown to be highly effective in attenuating acoustic reflections from vortical structures passing through the outlet boundary. The solid boundary on the "casing' was treated as a slip wall since simulation of the boundarylayer on the casing was not part of this investigation; the influence of the casing with 
relative motion between blade tip and casing wall is a subject for future investigation. The effect of casing shear on the tip flow was the subject of a recent experimental investigation in a rotating transonic high-pressure turbine by Jackson et al. (2015). In the study of Jackson et al. (2015), experimental results were compared with computational simulations where a no-slip wall and a slip-wall were imposed on the casing. The effect of casing shear was to change the tip-surface adiabatic wall temperature due to the effect of casing shear on the relative total temperature in the tip gap. Nonetheless, the flow field was qualitatively similar to cases without casing shear, in terms of the general flow structure and reattachment point heat transfer levels. For the present study it was decided to model the casing as a slip wall; this avoided the non-trivial task of imposing a mixed in-flow/out-flow boundary at gap exit (since the moving wall drags the endwall boundary-layer into the gap). Furthermore the nature of the endwall boundary-layer adds several more uncertainties which were beyond the scope of this study (aspects such as boundary-layer skew, thermal profile of the boundary-layer). Including the end-wall boundary layer would have been further complicated by the fact that the state of the endwall boundary-layer in an engine environment has so far not been widely reported and a 'correct' representation of this in itself is something beyond the scope of this study.

The tip surface was treated as a non-slip isothermal boundary. It should be noted that for non-adiabatic CFD calculations it is common to use an isothermal wall condition, as performed in this study, although the true wall temperature will depend on the heat transfer to the solid as well as the fluid, which requires a conjugate analysis. The Nusselt number, however, is normally considered to be independent of wall temperature (provided the wall temperature is not greatly varying). The solid walls in the upstream blocks were treated as slip walls apart from the vertical face on the right in block 1 (see figure 2). This wall represents the pressure-side surface of the blade and was either treated as a slip or no-slip wall depending on the test case, as discussed later. The additional slip wall case was considered in order to determine the importance of the pressure side boundary layer in affecting the global tip-gap flow. This was particularly important as it was felt that accurately representing the boundary layer characteristics of a full turbine configuration would be challenging at best.

The inlet flow was prescribed using the following relationships for the $u, v$ and $w$ components of velocity:

$$
\begin{gathered}
u=u_{i n}+\frac{4}{\pi} A\left(\cos \left(\theta_{y}\right)-\frac{\cos \left(3 \theta_{y}\right)}{3}+\frac{\cos \left(5 \theta_{y}\right)}{5}-\frac{\cos \left(7 \theta_{y}\right)}{7}\right) \\
\times\left(\cos \left(\theta_{z}\right)-\frac{\cos \left(3 \theta_{z}\right)}{3}+\frac{\cos \left(5 \theta_{z}\right)}{5}-\frac{\cos \left(7 \theta_{z}\right)}{7}\right) \\
v=A \sin \left(\theta_{z}\right) \cos \left(\theta_{y}\right) \sin (2 \pi t u / l) \\
w=w_{i n}+A \cos \left(\theta_{z}\right) \sin \left(\theta_{y}\right) \sin (2 \pi t u / l)
\end{gathered}
$$

where $u_{i n}$ and $w_{i n}$ are the spatial average of the $u$ and $w$ components of velocity over the inlet; $\theta_{y}=2 \pi y / l ; \theta_{z}=2 \pi z / l$. The length scale was $l=0.2 g$, and the amplitude $A$ was either $0,2.0$ or 4.0 depending on the case. This method approximates the flow downstream of a perforated sheet by producing a spatial 'chessboard' pattern in the $u$ velocity, and temporal fluctuations in $v$ and $w$ which simulate streamwise vortices convecting at the local velocity, whilst remaining divergence free.

These equations were used to generate deterministic perturbations at the inflow at low computational costs (other methods can be used but can be computationally expensive). This set of equations provided a way of generating fluctuations over a range of scales 


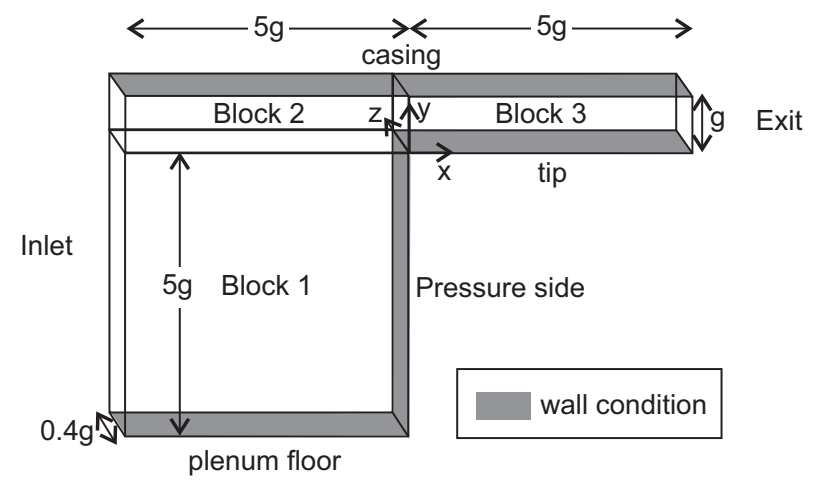

Figure 2. Computational domain

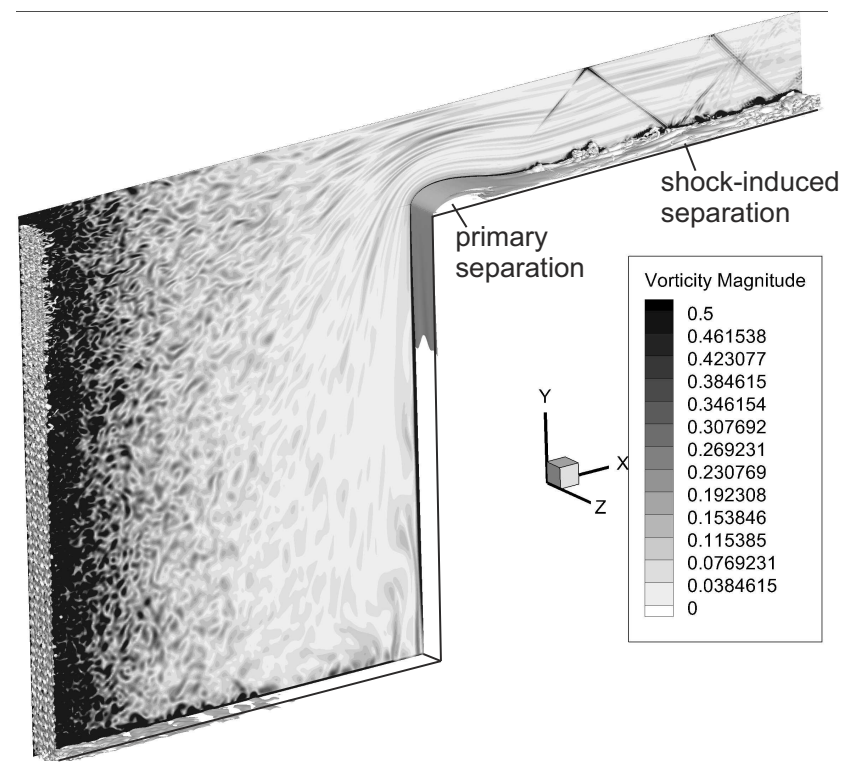

Figure 3. Example of simulated flow-field with turbulent inflow. Contours of vorticity magnitude and iso-surface of vorticity magnitude, $\omega=5$.

which stability analyses (shown later) suggested the tip flow shear layer would be sensitive to. Figure 3 shows an example flow-field with this inlet disturbance specification; it is interesting to note the large attenuation of turbulence and stretching of the vorticity field as the flow accelerates into the tip gap.

The turbulence levels and length-scales in the real turbine environment are not well known, especially near the endwalls where turbulence will arise from a wide range of sources such as endwall boundary-layers, secondary-flows and stator-rotor interactions. Thus the results presented in this paper give an indication of the turbulent flow within the tip gap when subjected to turbulence at high and low intensity, albeit with length scales of a similar order of magnitude as the tip gap.

\subsection{Overview of test cases}

A number of test cases were simulated to determine the sensitivity of the tip flow to free-stream disturbance, cross-flow and pressure-side boundary-layer (see table 1). The 'DATUM' case simulates a tip flow without cross-flow and with a clean inflow, i.e., with 
TABLE 1. Case details.

\begin{tabular}{clll}
\hline Case name & $w_{i n}$ & Tu at $x=-2.5, y=-2$ & PS \\
\hline DATUM & 0 & $0 \%$ & no-slip \\
XFLOW & 0.25 & $0 \%$ & slip \\
XFLOW+PS & 0.25 & $0 \%$ & no-slip \\
XFLOW+PS+TU & 0.25 & $1.0 \%$ & no-slip \\
XFLOW+PS+TU2 & 0.25 & $1.7 \%$ & no-slip \\
\hline
\end{tabular}

no free-stream disturbances or pressure-surface boundary-layer. The cases with cross flow ('XFLOW') all had a cross-flow of $25 \%$ of the tip-gap exit velocity. In the actual turbine tip flow, the ratio of cross-flow velocity to gap-exit velocity will largely be determined by the ratio of the pressure-side and suction-side velocities (since the gap-exit velocity will be similar to the local suction-side velocity and the cross-flow velocity will be set by the local pressure-side velocity). A value for this ratio of around 0.25 is thus representative of a tip-flow exiting near to peak suction. The 'XFLOW' and 'XFLOW+PS' cases both have zero amplitude inlet free-stream disturbances. However while the 'XFLOW' case sets a slip-wall for the pressure surface (PS), the 'XFLOW+PS' simulates the effect of a pressure surface boundary-layer by including the pressure surface (PS) as a no-slip wall. Introducing cross-flow makes it possible to test the effect of the pressure-side boundarylayer on the tip gap flow. The cases with a slip wall and with no-slip on the pressure-side wall are compared to each other in this study in order to determine whether the pressure side boundary-layer plays a significant role in the stability of the tip surface boundarylayer and separation.

The other two cases, 'XFLOW+PS+TU' and 'XFLOW+PS+TU2', include two levels of inflow disturbances, generated by the method discussed above. For these two cases wave amplitudes of $16 \%$ and $32 \%$ are prescribed at the inlet, respectively. Figure $4(\mathrm{a})$ shows the variation in turbulent kinetic energy within the plenum, averaged over the plenum height; there is a rapid reduction in turbulent kinetic energy over the first 10 grid points where high frequency energy is dissipated due to the non-physical nature of the introduced flow perturbations and filtering (since the square wave approach we use introduces both high and low frequency content). Further downstream, there is a more gradual reduction in turbulent kinetic energy, due to a combination of having relatively low speed and thus low Reynolds number flow upstream of the gap, with high viscous dissipation, and very strong acceleration when entering the gap. This means that the turbulence intensity at the inlet to the tip gap drops to below $1 \%$ despite the very high levels upstream. In table 1 we therefore quote the turbulence intensity at the center of the plenum.

The experimental results of Zhang et al. (2014) in a transonic turbine cascade have previously shown that tip heat transfer is insensitive to changes in turbulence intensities from $1-9 \%$, and this is likely a result of the observed attenuation of free-stream turbulence seen here. Power spectra of the free-stream disturbances at the inlet to the tip gap are shown in figure 4; the unsteadiness is largely distributed at low frequencies, and this is a result of specifying a relatively large length scale to the inlet disturbances $(l=0.2)$ as described earlier. 


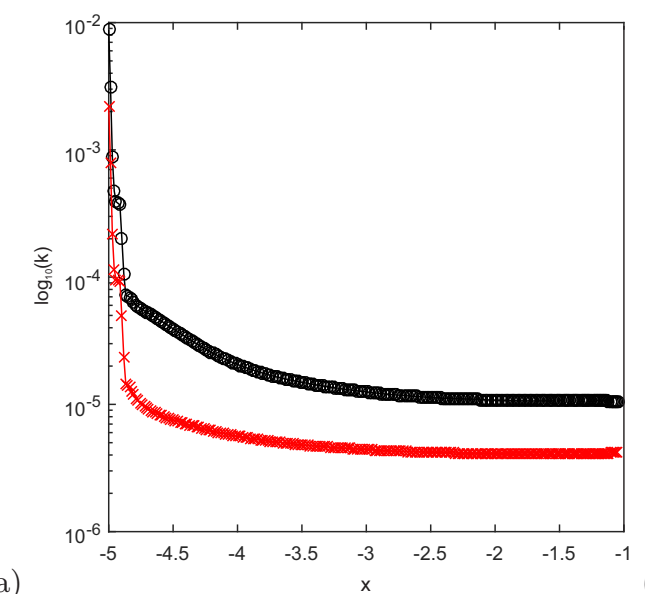

(b)

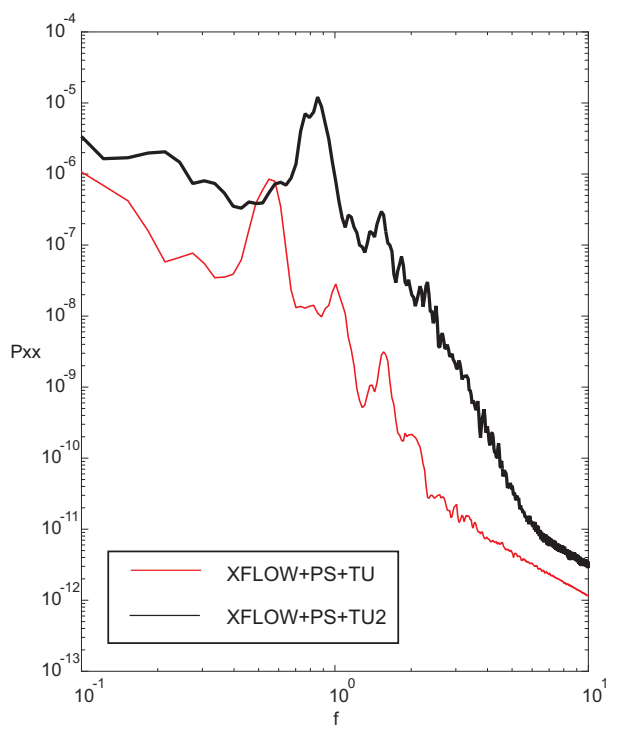

FIGURE 4. Variation of turbulent kinetic energy with distance upstream of tip-gap (left); Power spectra of velocity magnitude at gap inlet $x=0.0, y=0.7$ for two inlet disturbance levels (right).

\subsection{Grid sensitivity}

Figure 6 shows the computational mesh in the region of the inlet corner. Contours of vorticity magnitude are shown to indicate the shear layer of the separation bubble. The near-wall grid spacings in the $\mathrm{x}, \mathrm{y}$ and $\mathrm{z}$ directions were typically on average $y^{+}=0.4$, $x^{+}=2$ and $z^{+}=4$ over the tip surface for the case with 16 modes and spanwise extent of $0.4 \mathrm{~g}$. A mesh sensitivity study was performed at the datum conditions of $R e_{g}=20 \mathrm{~K}$, $w_{i n}=0, A=0$. Figure $7(\mathrm{a})$ shows time and spanwise averaged results of skin friction from three-dimensional calculations using the datum grid with a spanwise extent of $0.4 g$, and results using a finer grid which had a $50 \%$ increase in grid points in each direction in physical space (thus having over twice as many cells as the datum grid in the $x-y$ plane). The 'datum' mesh used here had 200 points across the height of the gap (in the $y$ direction), and 1000 points across the width of the gap (in the $x$-direction). Within the upstream plenum there were 400 points in the $x$ direction and 400 points in the $y$ direction. Results using either 16 and 32 Fourier modes across the span are also shown in figure $7 \mathrm{a}$ ). Data downstream of $4.75 \mathrm{~g}$ should be ignored because this is within the region of the zonal characteristic boundary condition. The figure shows little differences between the solutions over the majority of the tip $(\sim 1 \%)$, especially upstream of $3.5 \mathrm{~g}$. The region of sensitivity downstream of $3.5 \mathrm{~g}$ is due to the influence of a shock/boundarylayer interaction in this region, as will become evident later in the paper. The difference in predicted skin friction between the datum grid with 16 spanwise modes, and the fine grid with 32 spanwise modes peaks at about $10 \%$ within this region. The choice of at least 16 modes across a span of $0.4 \mathrm{~g}$ ensured that spanwise variations in the flow were adequately captured (i.e, there was at least a 100 fold reduction in the amplitude of the Fourier modes from the 1st to the 16th mode). The span is kept small in order to allow for a higher grid resolution in the spanwise direction. In Figure 7(b) skin friction results are compared for two cases with different spanwise extent $(0.4 g$ and $0.8 g)$. For these two cases, the number of modes per unit spanwise distance is preserved i.e., the number of modes is doubled from 32 to 64 when the spanwise extent is increased from 


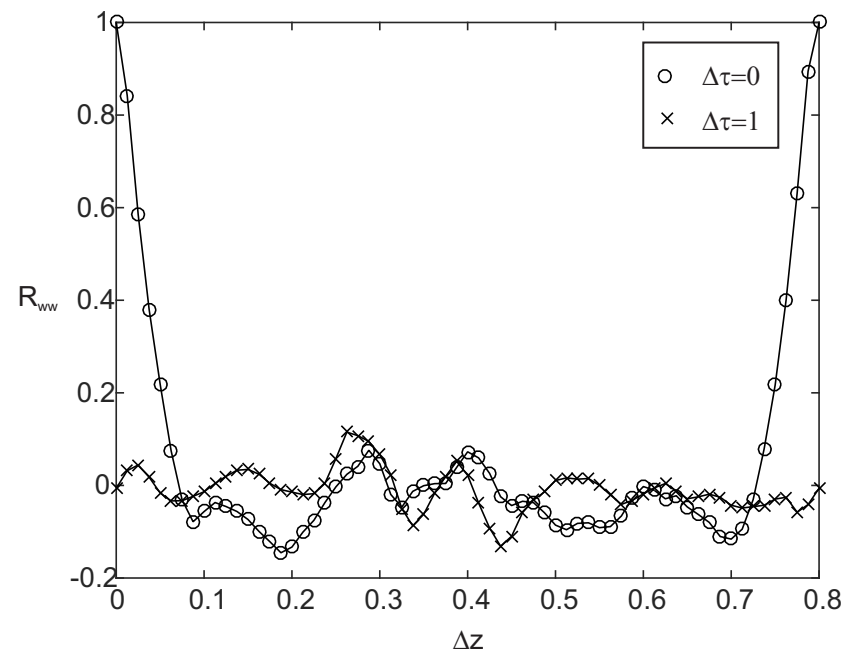

FiguRE 5. Auto-correlation function of spanwise velocity $w^{\prime}$ at $x=4.2, y=0.1,\left(w_{\text {in }}=0\right.$, $A=0, \mathrm{span}=0.8 g, 32$ spanwise modes)

$0.4 g$ to $0.8 g$. It can be seen that the wider domain has an almost negligible influence on the skin friction (see figure $7, \mathrm{~b}$ ). As will be shown in the section on stability analysis (section 4.1 ) a width of $0.4 \mathrm{~g}$ is sufficient to capture the most unstable spanwise modes that lead to breakdown to turbulence for the datum case.

Fourier spectra within the boundary-layer showed that fluctuations in turbulent kinetic energy are captured over 5 orders of magnitude using this datum mesh (see figure 8). It is interesting to note at this point the departure of the spectrum from the $-5 / 3$ slope characteristic of isotropic turbulence. Although this will be discussed in more detail later, it is useful to bear in mind that the tip flow does not undergo a complete transition to fully turbulent flow.

The auto-correlation function for the spanwise velocity component is given in Equation 3.4 , and this can be used to estimate the de-correlation of the flow with time and spanwise distance.

$$
R_{w w}(\Delta \tau, \Delta z)=\frac{\int_{0}^{\infty} w^{\prime}(t, z) w^{\prime}(t+\Delta \tau, z+\Delta z) d t}{\int_{0}^{\infty} w^{\prime}(t, z)^{2} d t}
$$

Figure 5 shows the variation of the auto-correlation function of the spanwise velocity component $R_{w w}(\Delta \tau, \Delta z)$ with spanwise distance at two phase differences $(\Delta \tau=0$ and $1)$. As expected, over large time shifts the flow becomes decorrelated from an initial state, as indicated by the low values of auto-correlation function at $\Delta \tau=1$. For the case when $\Delta \tau=0$, decorrelation occurs over spanwise distances greater than $0.1 \mathrm{~g}$, and this is further verification of the choice of $0.4 \mathrm{~g}$ as an appropriate spanwise extent for the DNS study conducted here.

\section{Results for the datum case}

We will begin in this section by discussing the datum case, where steady-state inflow was prescribed with no cross flow, and an inviscid/slip-wall was prescribed on the pressure-side. 


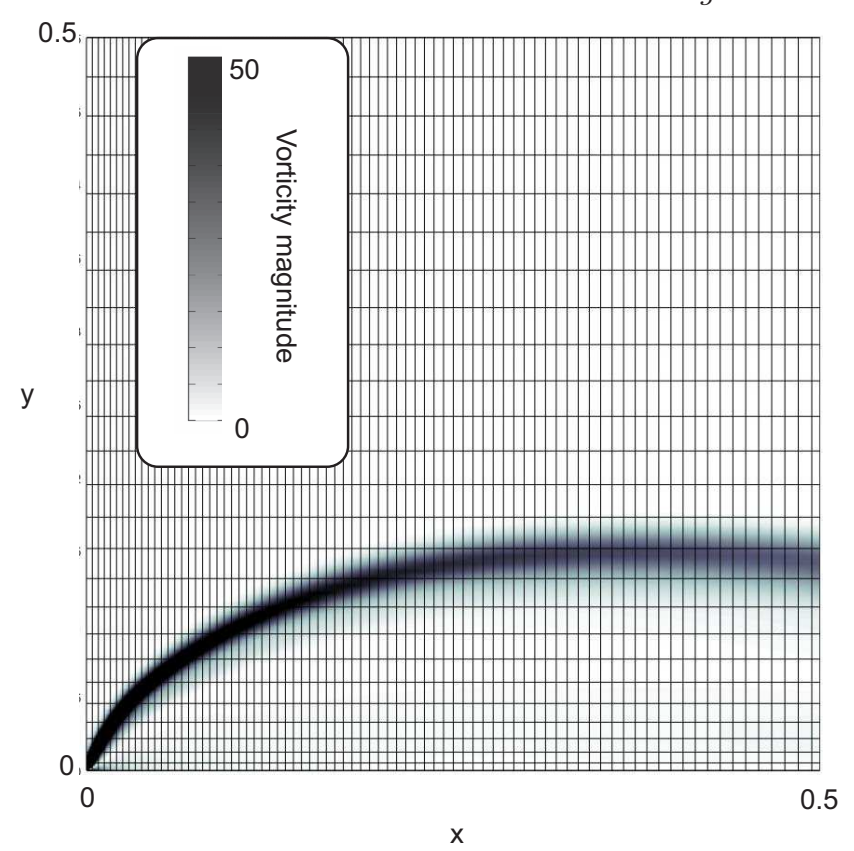

FIGURE 6. Computational mesh in the region of the separation bubble, showing only every $4^{\text {th }}$ grid-line.

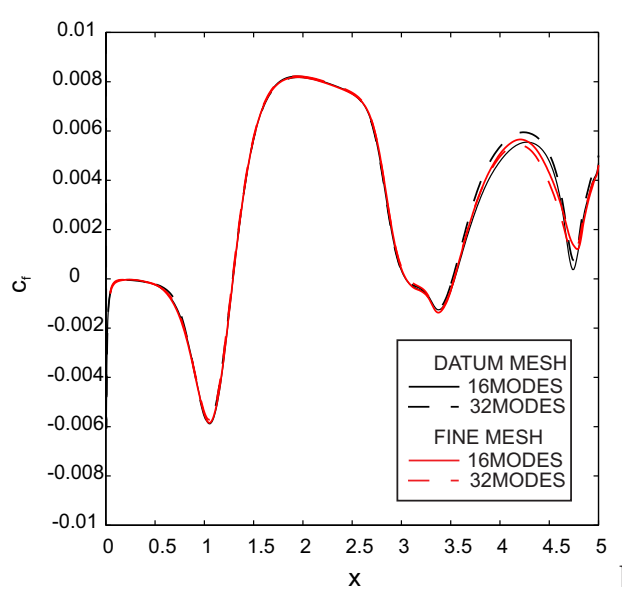

a)

FIGURE 7. Skin friction preditions for different grid sizes and spanwise extents.

\subsection{Stability analysis}

Initial two-dimensional solutions of the tip flow were found to not become unsteady. Figure 9 shows the predicted Mach number contours for the two-dimensional tip flow. The separation bubble at the inlet to the gap precedes the development of an oblique shock wave which reflects from the casing and goes on to interact with the tip boundarylayer, causing a shock-induced separation. The two-dimensional DNS results showed some small amplitude fluctuations in the flow field, but no unsteadiness was observed in either the primary or secondary separation (such as vortex shedding within the separated shear-layers). Constraining the spanwise extent of the tip flow to $0.1 \mathrm{~g}$ could produce a 


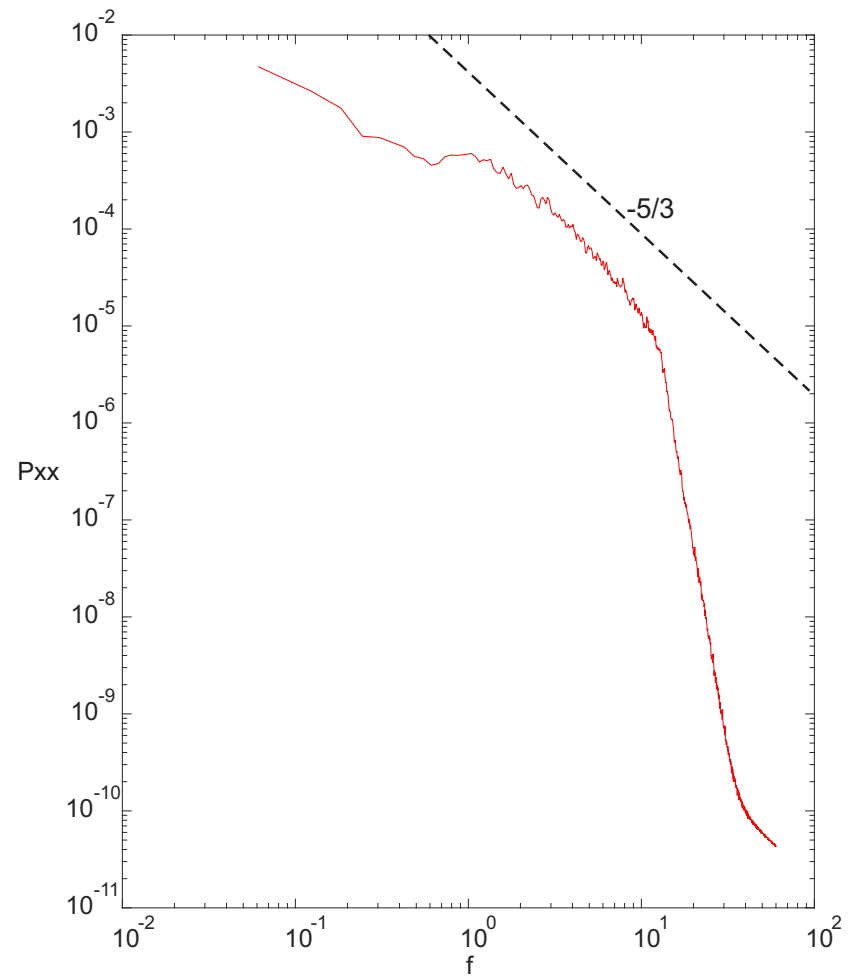

FIGURE 8. Power spectrum of velocity magnitude at $x=4.2, y=0.1\left(w_{\text {in }}=0, A=0\right.$, $\operatorname{span}=0.4 g$ ).

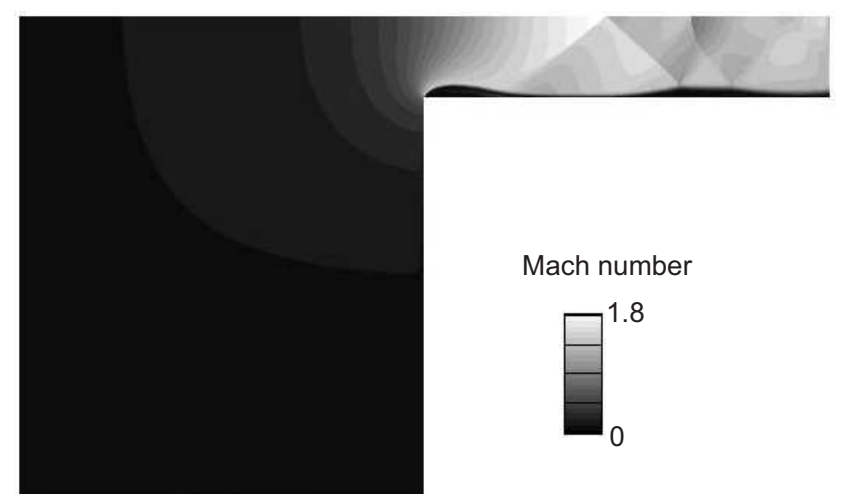

Figure 9. Example of simulated flow-field; Mach number contours from a solution of the two-dimensional tip flow $R e_{g}=15 \mathrm{~K}$ (datum grid).

similarly stable result when running three-dimensional simulations, even when the flow was subjected to a forced perturbation.

This implies that the flow is absolutely stable with respect to $2 \mathrm{D}$ perturbations and to 3D perturbations with a wavelength smaller than $0.1 \mathrm{~g}$. Although convective instabilities could also cause the flow to become unsteady, in the $2 \mathrm{D}$ simulations no perturbations are introduced into the domain and the fact that steady solutions are obtained also in the narrow domain 3D simulations suggests that the growth rates of the convective instabilities with small spanwise wavenumber are too small to allow for the flow to transition. 
The stability of the flow was investigated using a multi-dimensional linear stability approach in order to determine the spanwise extent required to achieve transition to turbulence within either the primary or secondary separation bubble. The two-dimensional solutions provided a steady base flow for the stability investigations. The multi-dimensional stability analysis is conducted by employing DNS with forcing terms, rather than solving a multi-dimensional eigenvalue problem. Using the converged solutions from two dimensional DNS as base flow, the forced DNS approach is equivalent to a linear stability analysis on a two dimensional base flow, and is not restricted to parallel flows. With this approach, the absolute and convective stability characteristics of a given base flow can be investigated similar to the study by Jones et al. (2010).

To perform the stability analysis, the two dimensional results serving as the base flow are read in as the initial condition. In order to maintain the initial condition, or base flow, forcing terms are added to the Navier-Stokes equations

$$
\frac{\partial Q}{\partial t}=R H S-R H S_{0}
$$

where $Q$ contains the conservative variables $(\rho, \rho u, \rho v, \rho w, \rho E)$. RHS denotes the righthand-side terms of the Navier-Stokes equations, containing all spatial derivatives, and $R H S_{0}$ are the right-hand-side terms of the base flow, i.e. the temporal derivative of the base flow variables $\left(\left.\frac{\partial Q}{\partial t}\right|_{t=0}\right)$.

Thus, at the start of each simulation the right-hand-side of the Navier-Stokes equations, containing all spatial derivatives of the basic state, is computed and stored in $R H S_{0}$. The simulation is then progressed, subtracting the stored forcing term at each Runge-Kutta substep. The result is that, assuming there is no change or perturbation to the base flow field, the initial condition can be maintained as a reference state, upon which the behavior of small perturbations can be investigated. This method has been successfully used for stability investigations of airfoil flows (Jones et al. 2010) and supersonic wakes (Sandberg 2012). It should be noted that this method can only consider discrete modes that depend on the choice of the spanwise domain size and the number of Fourier modes. For that reason, several stability investigations with varying spanwise domain size were conducted for each case and only the simulations exhibiting the largest growth rates are presented in the following.

The center of the initial pulse added to the higher spanwise Fourier modes was located within the first separation bubble at $(x, y)=(0.2,0.03)$. The perturbation takes the shape of a spatial step function with amplitude $1 \times 10^{-8}$ with three grid points in the streamwise and wall normal directions. This sharp-edged spatial and temporal distribution will excite a wide range of wavenumbers and frequencies at low amplitude. No further perturbations are introduced, and the response of the flow is monitored as the simulation is progressed. If the flow were convectively unstable, the initial perturbation would be expected to convect downstream whilst growing in amplitude, ultimately leaving the flow unperturbed. If the flow were absolutely unstable, the initial perturbation would be expected to grow exponentially in time until reaching non-linear amplitudes, at which point the forced DNS approach loses validity because the force terms will not be able to maintain the base flow. The response of the flow field is monitored by recording the time history of the perturbations at the forcing locations. In the context of a separation region behind a bluff body, it has previously been established that the pulse response, once an initial transient was overcome, was not sensitive to the location or shape of the pulse, given that the pulse location was within the recirculation region (Sandberg \& Fasel 2006).

The effect of perturbing the primary separation can be observed in figure 10 a) and b). The figures show the temporal evolution of the amplitudes of 8 spanwise Fourier 


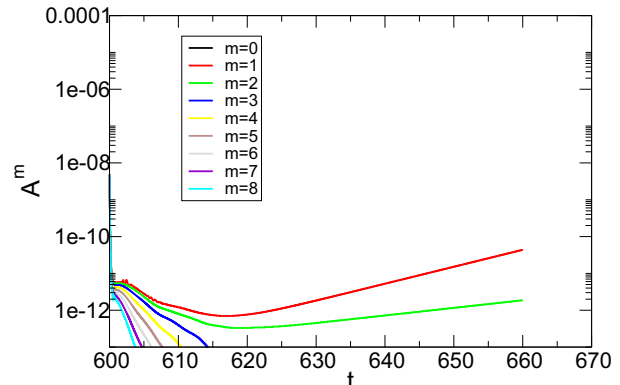

(a)

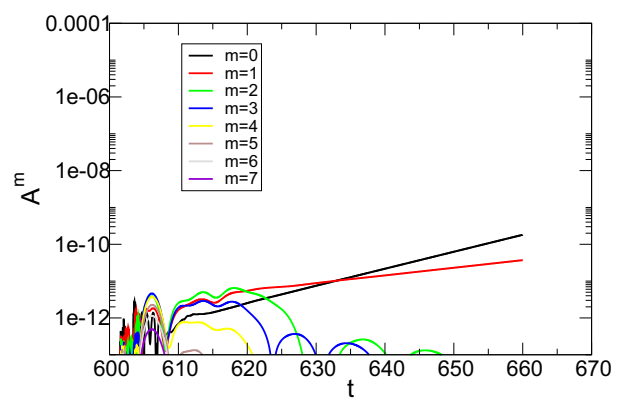

(c)

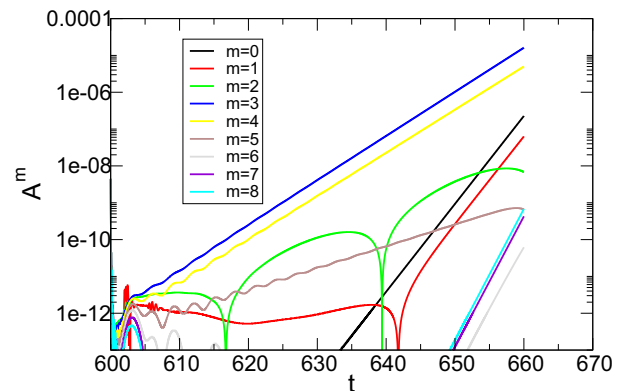

(b)

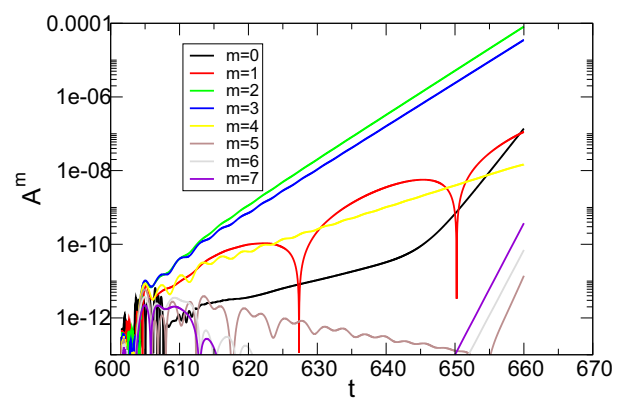

(d)

Figure 10. Stability of primary (top) and secondary (bottom) separation at $R e_{g}=1 \times 10^{4}$ (left) and $R e_{g}=15 \times 10^{3}$ (right); m=spanwise mode, span=0.8g.

modes within the primary separation bubble when the primary separation experiences a small amplitude pulse. A domain size of $0.8 \mathrm{~g}$ was used, so mode $m=1$ corresponds to an instability wave with spanwise wavelength of $0.8 \mathrm{~g}$. At $R e_{g}=1 \times 10^{4}$ the flow seems marginally unstable for the large wavelength modes and no growth in the higher modes can be observed, but increasing Reynolds number to $R e_{g}=15 \times 10^{3}$ leads to a clear development of an absolute instability. For the case when $R e_{g}=15 \times 10^{3}$, modes $\mathrm{m}=3$ and 4 appear to be the most linearly unstable initially (for the domain size investigated), and these correspond to spanwise wavelengths in the range 0.2-0.27. The other spanwise modes start growing later in time, due to non-linear interaction between initially linearly unstable modes.

The same procedure was performed perturbing the base flow in the secondary (shockinduced) separation. The stability of the second separation shows a similar behavior to that of the primary separation (see figures $10 \mathrm{c}$ and d). At $R e_{g}=15 \times 10^{3}$, modes $m=2$ and 3 appear to be the most unstable initially, providing further confirmation that a spanwise domain size of $0.4 \mathrm{~g}$ for the DNS is sufficient.

\subsection{Effect of Reynolds number}

The preceding section demonstrated the requirement for sufficient spanwise extent in order to allow for inclusion of the unstable spanwise modes that lead to the generation of intermittent three-dimensional structures further downstream. The following results were determined from three-dimensional DNS with a spanwise extent of $0.4 \mathrm{~g}$, which provides sufficient range to capture the most unstable three-dimensional modes (see above). The 


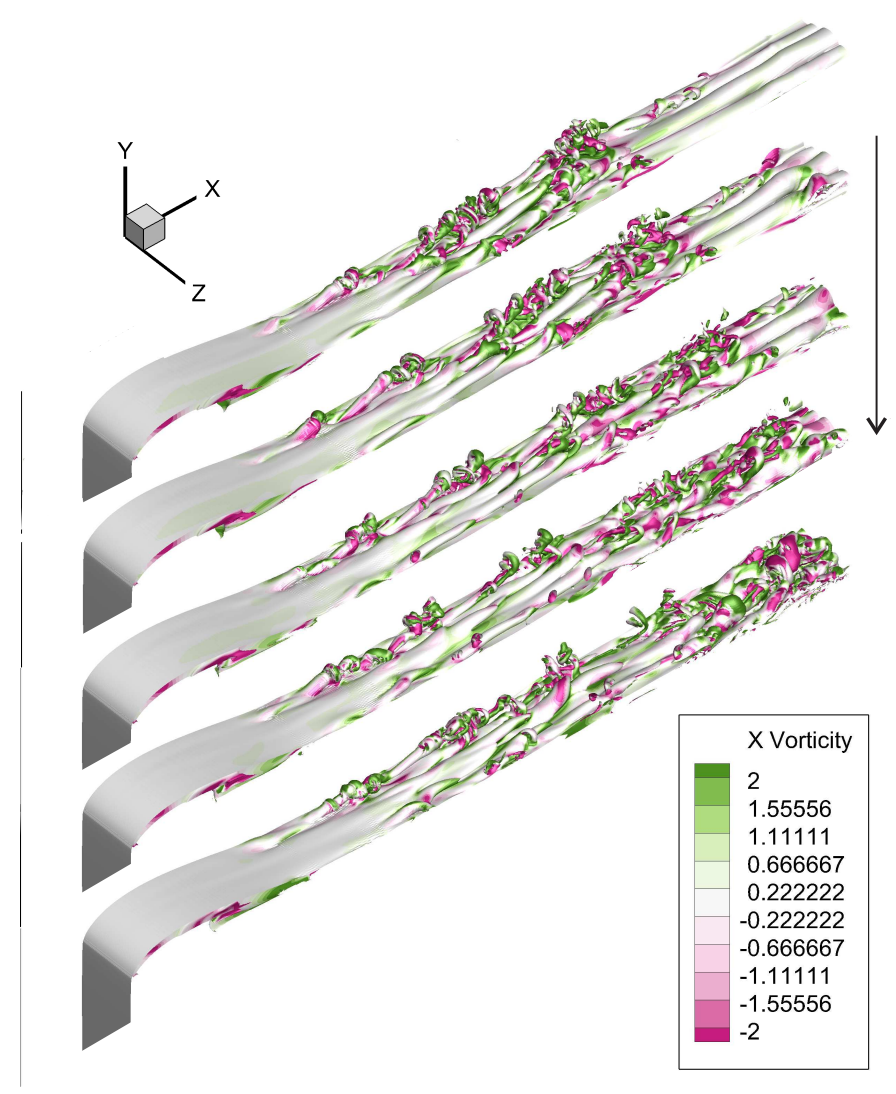

FIGURE 11. A sequence of instantaneous snapshots of iso-surfaces of vorticity magnitude $(\omega=5)$ spaced $\Delta t=0.5, R e_{g}=20 \times 10^{3}$ (fine grid, span $=0.4 g, 32$ spanwise modes).

effect of doubling the span from $0.4 \mathrm{~g}$ to $0.8 \mathrm{~g}$ was shown previously to have a small influence on the results (see Figure 7).

The turbulent structure of the flow can be observed in figure 11. This shows a sequence of instantaneous snapshots of vorticity isosurfaces. The shear-layer forming the separation bubble appears predominantly laminar, but downstream of the reattachment, hair-pin structures appear to develop. The legs of these structures have spanwise spacings around $0.15-0.2 g$, which agrees well with the previous stability analysis. For the time instants shown here, a patch of turbulent flow is seen propagating downstream, but following and preceding this is a region of relatively steady flow (i.e., possessing hardly any smaller scale structures).

The influence of Reynolds number on the tip flow structure can be observed in figure 12 . The most apparent effect of Reynolds number is the change to the secondary separation and shock structure. As the Reynolds number rises the height of the secondary separation is reduced and the second shock/boundary-layer interaction subsequently shifts downstream. The effect of Reynolds number on the surface quantities is shown in figure 13 . The influence on the tip surface pressure (identified by the isentropic Mach number) is small in the region of the inlet separation bubble, reattachment region (marked R), and supersonic acceleration downstream of the bubble. This suggests the bubble shape is 

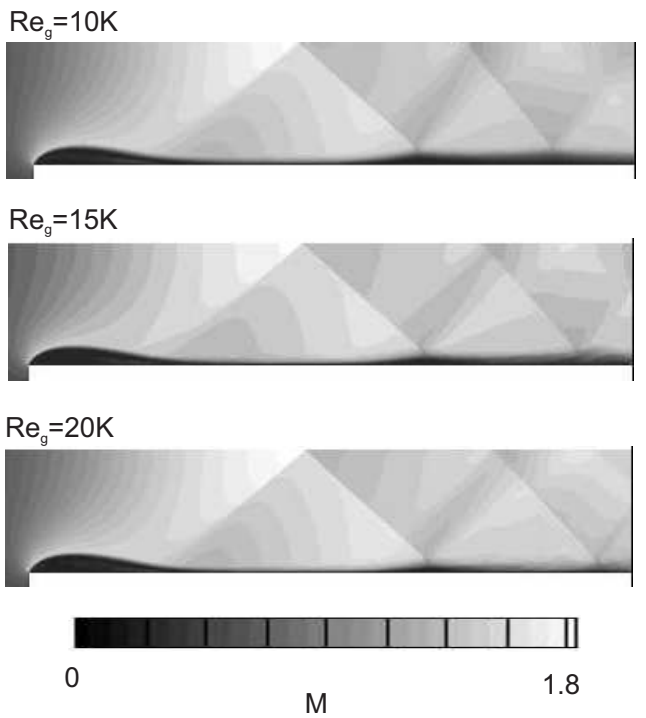

Figure 12. Spanwise average instantaneous Mach number contours from 3D solutions at various Reynolds numbers, span $=0.4 g$ (datum grid, 16 spanwise modes).
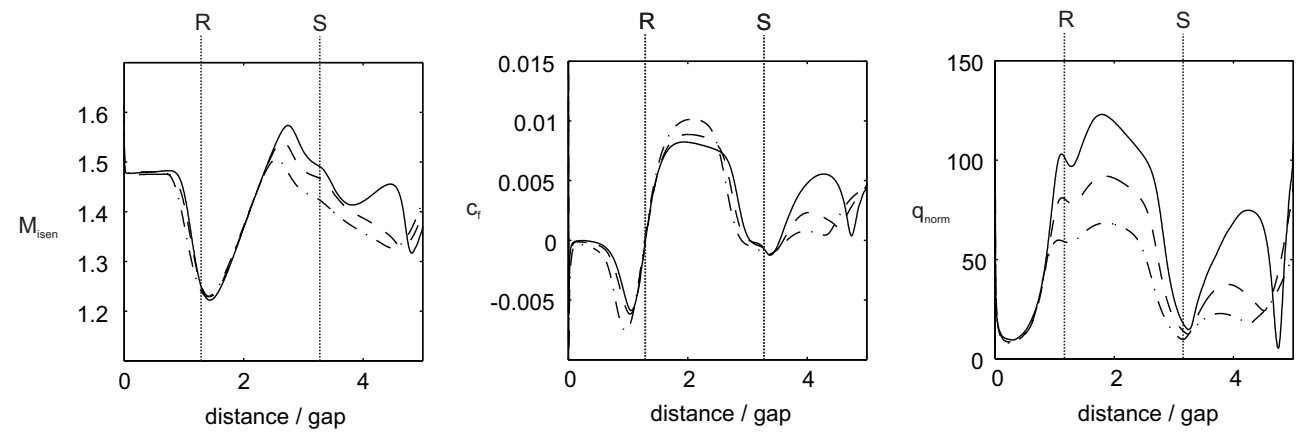

FIGURE 13. Surface isentropic Mach number, skin friction and normalized heat flux on the tip surface for : $R e_{g}=20 \times 10^{3}(-) ; R e_{g}=15 \times 10^{3}(---) ; R e_{g}=10 \times 10^{3}(-\cdot-)$; span $=0.4 g$

largely insensitive to Reynolds number, since the shape of the primary separation bubble controls the pressure field in this region. In contrast, near the first shock/boundary-layer interaction (marked S), the influence of Reynolds number on the local surface pressure field is significant; as the secondary separation is reduced at higher Reynolds numbers, the free-stream Mach number rises. For boundary-layers which are not undergoing transition, the magnitude of the skin friction typically reduces as Reynolds number is increased. The results upstream of $x / g=3$ in figure 13 are consistent with this. Downstream of $x / g=3$, and particularly downstream of the shock/boundary-layer interaction $(\mathrm{S})$, the skin friction rises as Reynolds number is increased. This is because the flow is not fully turbulent, but is in the process of transition; a process which is augmented by increasing the Reynolds number and by the presence of the shock/boundary-layer interaction. This has a significant effect on the heat-transfer in this region as also shown in figure 13 . The figure shows that upstream of $\mathrm{S}$, the rise in $q_{\text {norm }}$ is consistent with the rise in Reynolds number (doubling $R e_{g}$ leads to about an $80 \%$ increase in $q_{\text {norm }}$ at $x / g=2$ ). Downstream of $\mathrm{S}$, the effect of doubling the Reynolds number has a proportionately much greater effect; doubling $R e_{g}$ leads to a 3.5 fold increase in $q_{\text {norm }}$ at $x / g=4$. 


\subsection{Heat transfer coefficient, Adiabatic Wall Temperature and Recovery coefficient}

An additional simulation of the $R e_{g}=20 \times 10^{3}$ case described above was performed at a reduced wall temperature of $T_{w}=0.89 T_{o}$, and the difference in wall heat flux between the two cases was used to determine the heat transfer coefficient, adiabatic wall temperature and recovery factor. For convective heat transfer, it is conventionally assumed that the surface heat flux is proportional to a heat transfer coefficient and a driving temperature difference:

$$
q=h\left(T_{a w}-T_{w}\right)
$$

where $T_{a w}$ is the adiabatic wall temperature (equal to the recovery temperature). Under the assumption that the heat transfer coefficient $(h)$ and adiabatic wall temperature are not dependent on $T_{w}$ over the range of gas-to-wall temperature ratios tested here (in this case $T_{w} / T_{o}$ is changed by $15 \%$ ), calculations at two different wall temperatures can be used to determine $T_{a w}$ and $h$; Equation 4.2 has two unknowns $T_{a w}$ and $h$, and therefore these can be determined using results of the computed surface heat flux at two wall temperatures.

The variation in heat transfer coefficient over the tip surface is shown in figure 14 . Also shown are the position of the primary separation bubble reattachment $(\mathrm{R})$ and shock/boundary-layer interaction $(\mathrm{S})$ as discussed in the previous section. The heat transfer coefficient varies greatly over the tip width, with a minimum value within the primary separation zone of $N u=15$ to a maximum at the reattachment point $(\mathrm{R})$ of $N u=95$. The effect of the shock/boundary-layer interaction at S, which leads to the secondary separation, also causes $N u$ to drop to around 15 although the following reattachmentpoint heat transfer coefficient peaks at a lower level of around $N u=50$, as compared to the primary separation point reattachment.

It is interesting to study how the presence of large regions of separated flow and strong free-stream pressure gradients affect the correlation between skin friction and heat transfer coefficient. A Reynolds analogy assumption would indicate that the Stanton number $\left(S t=h / c_{p} \rho_{\infty} u_{\infty}\right)$ and skin friction coefficient are directly proportional to each other, i.e. $c_{f}=2 S t$. Figure 14 compares $c_{f}$ with $2 S t$ over the tip surface. The Reynolds analogy is clearly not adequate over the majority of the tip surface. This departure occurs in regions of separation since, although the skin friction falls to zero, heat transfer will still be possible if there is a driving temperature difference, as shown by Spalart \& Coleman (1997) and Spalart \& Strelets (2000). This is most pronounced at the reattachment point $\mathrm{R}$, where the skin friction is zero and the heat transfer coefficient is at a maximum. The reattachment point heat transfer is largely driven by turbulence production in this region, and we will discuss this more in the next section. In general the heat transfer coefficient levels are significantly greater than one would expect from the simple Reynolds analogy. The results show that estimations of tip heat transfer using a Reynolds analogy will significantly underestimate tip heat load, and this is important from a turbine design perspective.

The variation in adiabatic wall temperature is shown in figure 15. As discussed earlier, data downstream of $x / g=4.75$ should be ignored due to the presence of the zonal characteristic condition in this region, and the spike in adiabatic wall temperature in this region is an artefact of this. Between $R$ and $S$, the variation in adiabatic wall temperature is consistent with the distribution of free-stream Mach number over the tip surface; the lowest value of $T_{a w}$ occurs in the region of peak free-stream Mach number.

The recovery factor $\left(r=\left(T_{a w}-T_{\infty}\right) /\left(\left(T_{o}-T_{\infty}\right)\right)\right.$ is also shown in figure $15 ; r$ displays quite a considerable variation over the tip from 0.75 to 0.95 between $x=0$ and 4.75 ; low values of $r$ occur within the separated regions, and also at the point of peak isentropic 

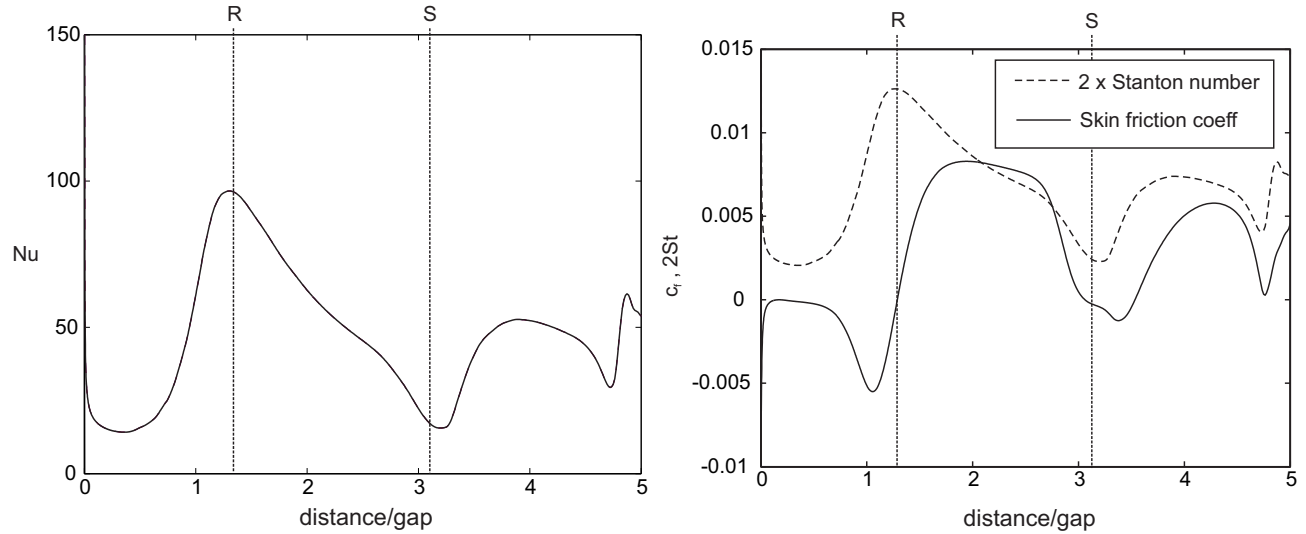

FigURE 14. Variation of heat transfer coefficient expressed as a Nusselt number (left) and comparison of Stanton number with skin friction coefficient (right), $R e_{g}=20 \times 10^{3}$, span $=0.4 \mathrm{~g}$
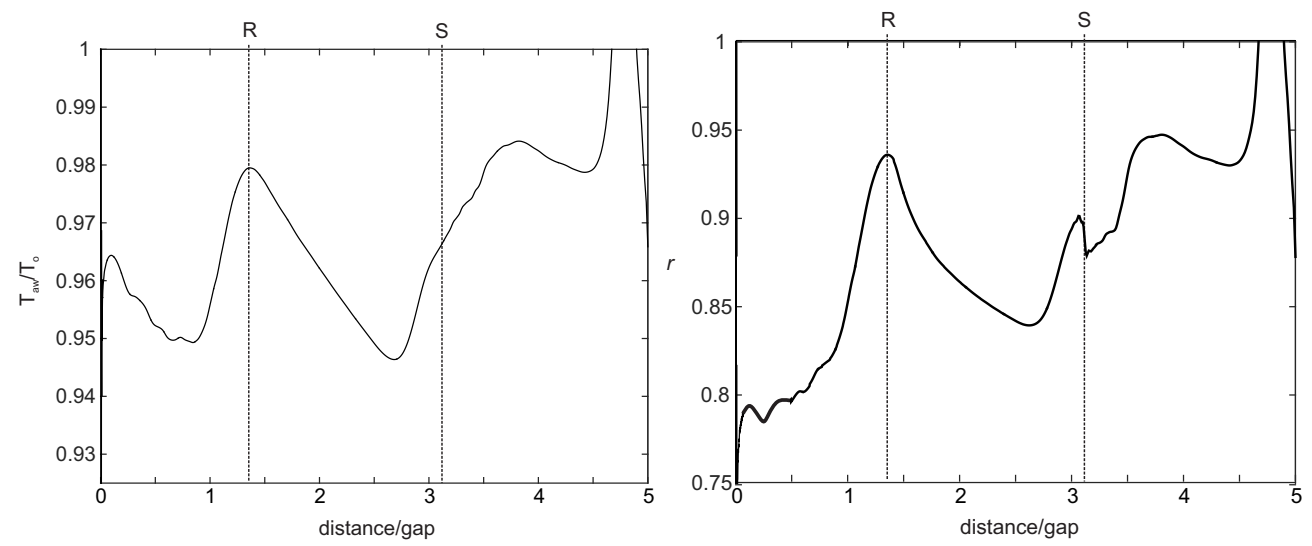

Figure 15. Variation of Adiabatic Wall Temperature (Recovery Temperature) (left) and Recovery factor $r$ (right), $R e_{g}=20 \times 10^{3}$, span $=0.4 \mathrm{~g}$

Mach number, while peaks in $r$ occur within the reattachment zones of both the primary and secondary separation bubbles. Typically values of recovery factor of between 0.83 to 0.9 are assumed, depending on the expected boundary-layer state and Prandtl number; for two-dimensional self-similar laminar boundary-layers the recovery factor can be shown to be approximately equal to $\operatorname{Pr}^{1 / 2}$ and thus for air $r \approx 0.85$ (see, for example, Kays $\&$ Crawford 1980). The results shown here are consistent with the range of values of recovery factor expected for laminar and turbulent boundary-layers. There is about a $20 \%$ variation in recovery factor over the tip surface with higher values of recovery factor in regions of high turbulent kinetic energy (as shown later in Figure 16).

\section{Effect of free-stream disturbances, cross-flow and pressure-surface boundary-layer on the tip flow}

Time-averaged Mach number and turbulent kinetic energy for the additional DNS performed are shown in figures 16. Comparing the 'DATUM' and 'XFLOW' cases shows that the effect of cross-flow appears to have little effect on the size and shape of the primary separation bubble, although the levels of turbulent kinetic energy produced in the primary separation are reduced by around $30 \%$ in the reattachment region with the 

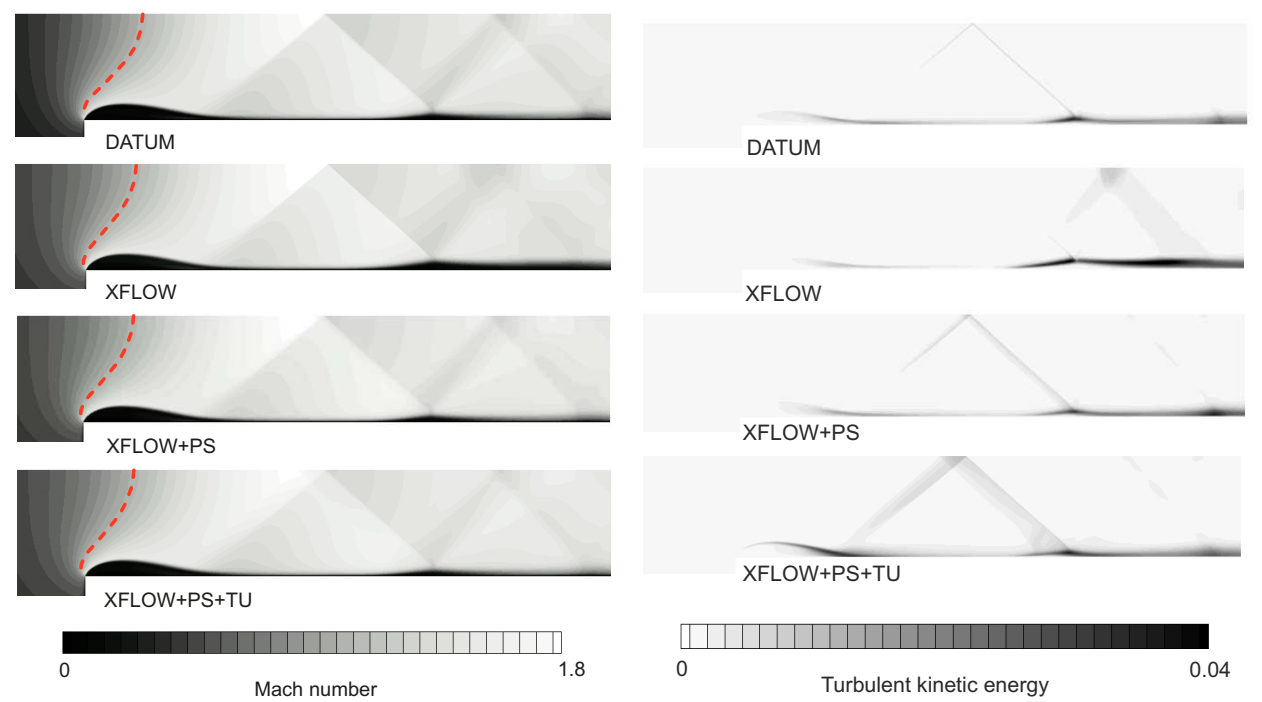

Figure 16. Time-averaged Mach number (left) and turbulent kinetic energy (right) for DNS test-cases (see Table 1); Dashed line indicates $M=1$ contour $\left(R e_{g}=20 \times 10^{3}\right.$, span $\left.=0.4 \mathrm{~g}\right)$.

addition of cross-flow; the reason for this is that cross-flow tends to stabilize the tip flow as will be shown in the stability analysis for the 'XFLOW' cases discussed later. The lower levels of turbulence produced in the 'XFLOW' case means that the boundary-layer is less able to withstand the downstream shock/boundary-layer interaction, and this leads to a much longer region of separated flow downstream of the shock impingement. These effects lead to a significant reduction in both the skin-friction and Nusselt number over the tip surface (see figure 17).

Figure 18 compares the time-averaged boundary-layer profile on the pressure-side from the XFLOW+PS case with the pressure surface boundary layer predicted in the DNS simulations of a transonic high-pressure turbine vane shown in Wheeler et al. (2016). The Reynolds and Mach numbers at vane exit for this study were $0.6 \times 10^{6}$ and 0.9 respectively. There is a surprisingly good agreement with the profiles, particularly comparing the vane pressure-surface boundary-layer profile at 20\% axial chord. Figure 19 compares the pressure-side boundary layer profile and turbulent kinetic energy for the cross-flow cases, showing that as the inflow disturbance amplitude is increased there is a significant increase in the turbulence intensity in the boundary layer, but the velocity profile itself does not greatly vary between the cases.

The effect of the pressure-surface boundary-layer can be observed by comparing the 'XFLOW' and 'XFLOW+PS' cases; figures 17 show that the presence of the pressuresurface boundary-layer leads to an increase in the production of turbulent kinetic energy in the primary separation, which consequently raises the skin-friction and Nusselt number and this offsets considerably the opposing stabilizing influence of cross-flow. The reattachment point of the primary separation bubble is not greatly affected by either cross-flow or the pressure-surface boundary-layer. In contrast, the secondary shock-induced separation is significantly influenced by both cross flow and pressure-surface boundary-layer; the stabilizing effect of cross-flow causes the secondary separation to remain unattached leading to low skin friction and heat transfer while the de-stabilizing effect of the pressuresurface boundary-layer causes a stronger breakdown to turbulence in the 'XFLOW+PS' case which then causes an earlier re-attachment of the secondary separation bubble. By comparing 'XFLOW+PS' to 'XFLOW+PS+TU' and 'XFLOW+PS+TU2' in figure 17, 

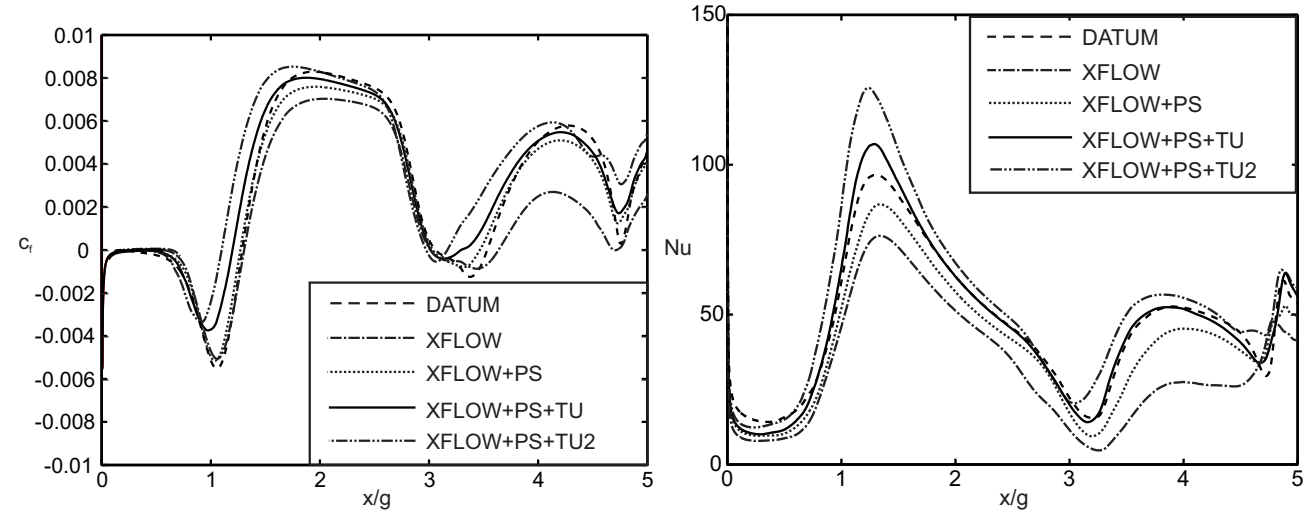

FiguRE 17. Time-average skin friction and Nusselt number for DNS test-cases (see Table 1).

- Current study (XFLOW + PS)

— PS boundary-layer at 20\% axial chord [Wheeler et al 2016]

-.... PS boundary-layer at $85 \%$ axial chord [Wheeler et al 2016]

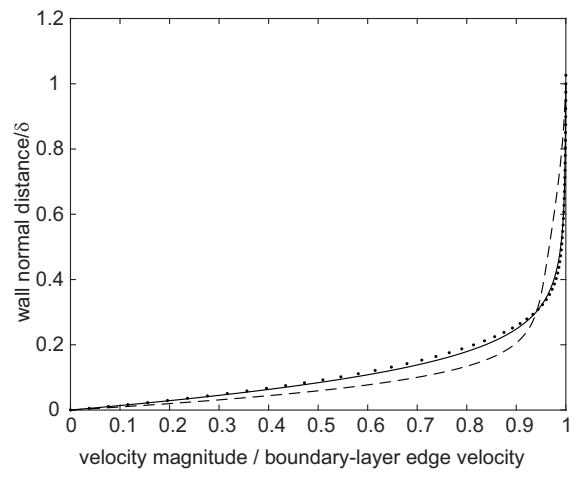

FiguRE 18. Boundary-layer profiles on the pressure side at $y=-1.0 \mathrm{~g}$ compared to pressure-side boundary-layers from HP vane calculations of Wheeler et al. (2016)

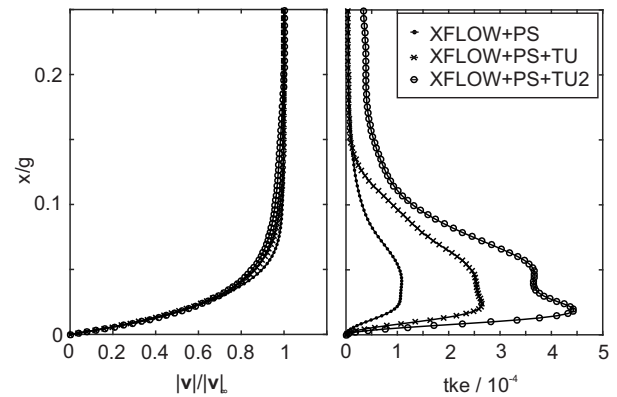

FiguRE 19. Boundary-layer profiles on the pressure side at $y=-1.0 g$

we see that the effect of free-stream disturbances is to raise the skin-friction and Nusselt number fairly uniformly over the tip surface. The free-stream disturbances also brings about earlier reattachment of both the primary and secondary separation bubbles.

An example of the instantaneous tip-gap flow at five sequential time instants is shown in figure 20 for the 'XFLOW+PS+TU' case, and this shows an intermittently turbulent flow-field. The turbulent structure of the flow in all cases was observed to be similar to that of the 'DATUM' case; the shear-layer forming the separation bubble appears 


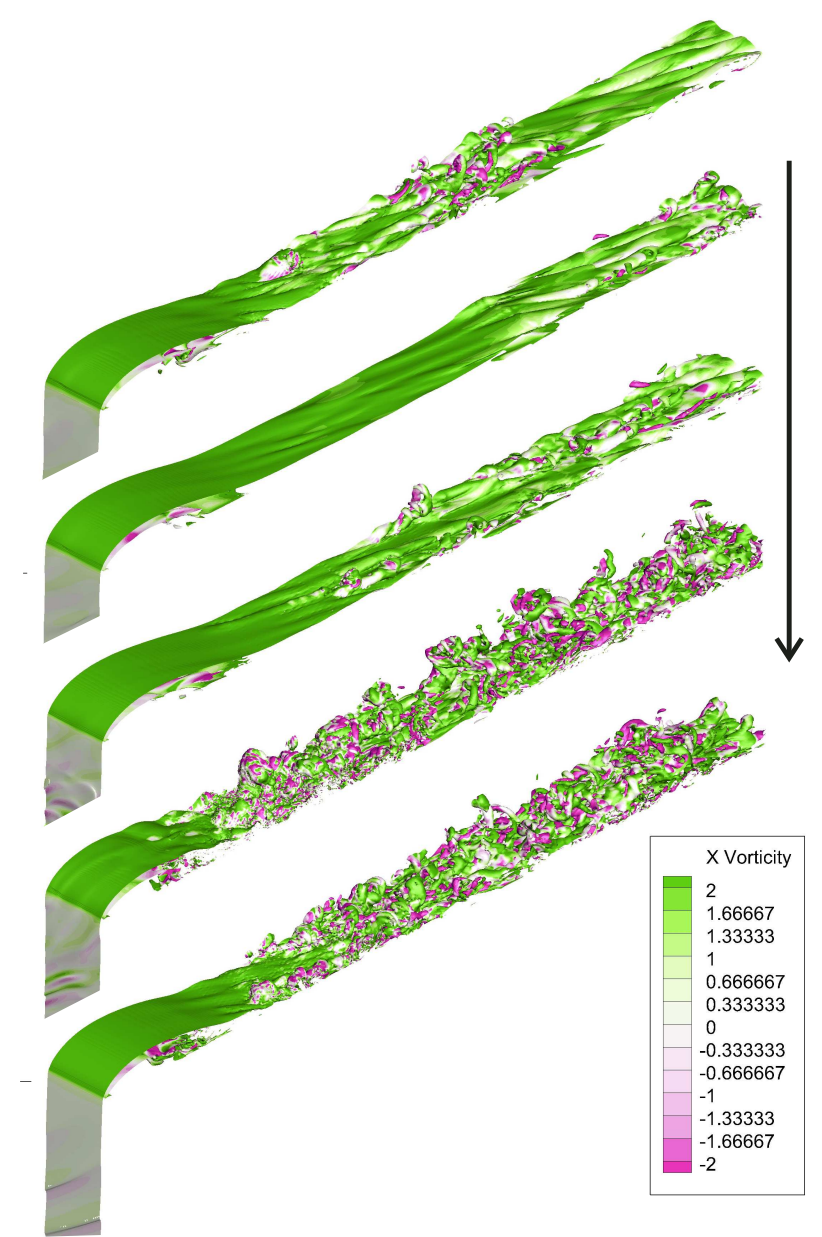

FiguRE 20. Instantaneous snapshots showing iso-surfaces of vorticity magnitude $(\omega=5)$ for the 'XFLOW+PS+TU' case; time between snapshots $\Delta t=9.6$

predominantly laminar, but downstream of the separation, hair-pin structures emerge. As in the 'DATUM' case, the legs of these structures have a spacing around $0.15-0.2 g$. In the penultimate image, there also appears to be structures propagating up the pressuresurface and into the tip-gap affecting the separation bubble and bringing about a rapid breakdown to turbulence directly.

\subsection{Stability analysis with crossflow}

We reported on the stability of the 'DATUM' case above and showed that breakdown to turbulence came about due to an absolute instability of the primary separation bubble. A stability analysis for the cross-flow cases ('XFLOW' and 'XFLOW+PS') are now discussed. As described above, the multi-dimensional stability analysis is conducted by employing DNS with forcing terms, rather than solving a multi-dimensional eigenvalue problem.

The effect of perturbing the primary separations in the 'XFLOW' and 'XFLOW+PS' cases can be observed in figure 21 . This figure shows the temporal evolution of the amplitudes of 8 spanwise Fourier modes within the primary separation bubble when the primary separation experiences a small amplitude pulse. For both cases a domain size of 
$1.5 \mathrm{~g}$ was used. It can be observed that the most unstable mode for the spanwise domain size investigated is $m=2$ in both cases, which corresponds to an instability wave with spanwise wavelength of $0.75 \mathrm{~g}$. As also observed in the 'DATUM' case, the separation is absolutely unstable, however the instability growth rates are reminiscent of those found at $R e_{g}=1 \times 10^{4}$ without cross-flow. In the original analysis for the 'DATUM' case, it was found that the growth rates were reduced at lower Reynolds numbers, and thus the effect of cross-flow is similar to reducing the tip Reynolds number. The introduction of cross-flow also leads to a shift to lower instability modes and thus larger wavelengths from $0.25 \mathrm{~g}$ ('DATUM') to $0.75 \mathrm{~g}$ for both cross-flow cases shown here; earlier stability analyses by Sandberg (2012) showed a shift to lower instability modes can occur when including eddy viscosity in the stability analysis, which in essence also reduces the effective Reynolds number. Previous work on separation in cross-flow on swept aerofoils (such as Horton 1968) have suggested that the stability of the separation can be determined from the Reynolds number resolved in the chordwise direction (i.e., the direction orthogonal to the leading-edge). This theory of independence of the chord-wise and cross-flow boundary-layers (Horton 1968) would suggest that for the same streamwise Reynolds number, the addition of cross-flow would reduce the chord-wise Reynolds number and thus we would expect an increased region of laminar flow with cross-flow. For our case the Reynolds number based on the $u$ component of velocity is about $4 \%$ lower than the streamwise Reynolds number (see section 2), and is unlikely to explain fully the increased stability of the cross-flow cases.

Another explanation is that the addition of cross-flow reduces the magnitude of the reverse flow within the separation bubble, and evidence for this is seen in the skin-friction data shown in figure 17. This is also shown in Figure 22 which shows the variation of the $x$-component of velocity at two positions in the separation bubble $(x=0.26 g$ and $x=0.57 \mathrm{~g})$ for the 'DATUM' and 'XFLOW' cases; the profiles show that the effect of cross-flow is to reduce the magnitude of the reverse flow in the near wall region $(y<0.04 g)$.

An additional DNS crossflow case was performed to determine whether the spanwise extent of $0.4 \mathrm{~g}$ was sufficient for the crossflow cases, given the shift to lower instability modes observed in the stability analysis discussed above. Figure 23 shows the predicted skin friction for the 'XFLOW' case with spanwise extents of 0.4 and $0.8 g$; the spanwise resolution was kept constant by doubling the number of spanwise modes for the case with $0.8 g$ spanwise extent. Skin friction in the region of reattachment downstream of the secondary separation is modified by the increased spanwise extent. Nonetheless there is little differences in the skin friction in the region of the primary separation bubble and first reattachment point, as well as the position of the secondary separation, indicating that even for the crossflow cases, a spanwise extent of $0.4 \mathrm{~g}$ is sufficient for capturing the correct boundary-layer state over the majority of the tip width.

\section{Conclusion}

Direct numerical simulations were conducted to investigate the unsteady flow over a model turbine blade-tip at engine scale Reynolds and Mach numbers. The particular case of a transonic tip flow was studied since previous work had suggested compressibility has an important influence on the turbulent nature of the separation bubble at the inlet to the gap and subsequent flow reattachment. The flow was simulated over an idealized tip geometry where the tip gap was represented by a constant area channel with a sharp inlet corner to represent the pressure side edge of the turbine blade. We investigated the influence of free-stream disturbances, cross-flow and pressure-side boundary-layer on 

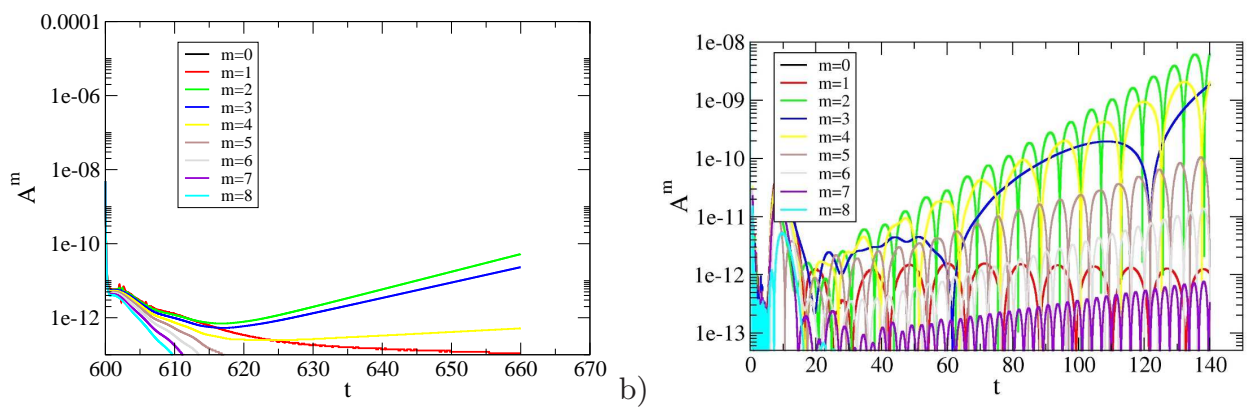

Figure 21. Growth of instability waves for: a) the 'XFLOW' case; b) the 'XFLOW+PS' case. (Spanwise extent $=1.5 \mathrm{~g})$.

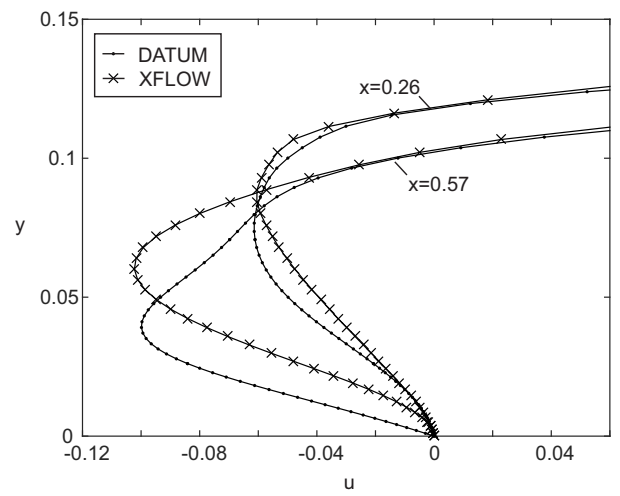

Figure 22. Profile of reversed flow in separation bubble for the 'DATUM' and the 'XFLOW' cases at $x=0.26 g$ and $x=0.57 g$

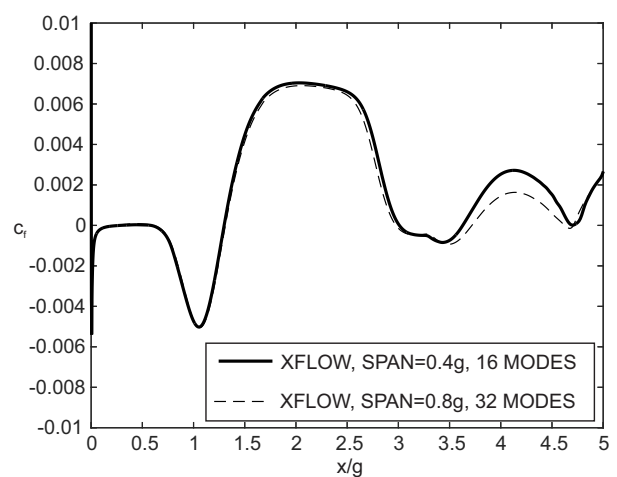

FiguRE 23. Skin friction predictions for the 'XFLOW' case with different spanwise extents.

the tip flow aerodynamics and heat transfer. For 'clean' in-flow cases we find that even at engine scale Reynolds numbers the tip flow is intermittent in nature (neither steady nor fully turbulent). The breakdown to turbulence occurs through the development of spanwise streaks with spacing around $15-20 \%$ of the gap height. Linear stability analysis of steady two-dimensional Navier-Stokes solutions of the same set-up found the most unstable global modes to have spanwise wavelengths around $25 \%$ of the gap height. Cross-flows of $25 \%$ of the gap exit velocity were found to increase the stability of the 
tip flow, and to significantly reduce the turbulence production in the separation bubble. This was also predicted through the linear stability analysis. For the case when the inlet flow contained free-stream disturbances, viscous dissipation and the rapid acceleration of the flow at the inlet to the tip-gap caused significant distortion of the vorticity field and reduction of turbulence intensity as the flow entered the tip gap. It was also shown that the Reynolds analogy generally does not hold, in particular in regions where skin friction approaches zero while a significant temperature gradient is present, such as at the reattachment point of the tip flow. Furthermore, the DNS results indicate up to $20 \%$ variation in recovery factor over the tip surface suggesting that the common assumption of constant recovery factor is inaccurate.

\section{Acknowledgments}

Computer time was provided by the UK turbulence consortium under EPSRC grant EP/L000261/1. This work was also supported by resources provided by The Pawsey Supercomputing Centre with funding from the Australian Government and the Government of Western Australia.

\section{REFERENCES}

Bunker, R. S. 2004 Blade Tip Heat Transfer and Cooling Techniques. VKI Lecture Series 2004-02 .

Carpenter, M. H., Nordstrm, J. \& Gottlieb, D. 1999 A Stable and Conservative Interface Treatment of Arbitrary Spatial Accuracy. Journal of Computational Physics 148 (2), 341.

Chen, G., Dawes, W. N. \& Hodson, H. P. 1993 A Numerical and Experimental Investigation of Turbine Tip Gap Flow. 29th Joint Propulsion Conference and Exhibit, AIAA 93-2253 .

Coull, J. D., Atkins, N. R. \& Hodson, H. P. 2014 Winglets for Improved Aerothermal Performance of High Pressure Turbines. Journal of Turbomachinery 136, 091007.

De Maesschalck, C., Lavagnoli, S. \& Paniagua, G. 2014 Blade Tip Shape Optimization for Enhanced Turbine Aerothermal Performance. Journal of Turbomachinery 136, 041016.

De Maesschalck, C., Lavagnoli, S. \& Paniagua, G. 2015 Blade Tip Carving Effects on the Aerothermal Performance of a Transonic Turbine. Journal of Turbomachinery 137, 021005 .

Harvey, N. W. 2004 Aerothermal Implications of Shroudless and Shrouded Blades. VKI Lecture Series 2004-02.

Horton, H. 1968 Laminar Separation Bubbles in Two and Three Dimensional Incompressible Flow. PhD thesis, Department of Aeronautical Engineering, Queen Mary College, University of London.

Jackson, A. J., Wheeler, A. P. S. \& Ainsworth, R. W. 2015 An Experimental and Computational Study of Tip Clearance Effects on a Transonic Turbine Stage. International Journal of Heat and Fluid Flow 56, 335-343.

Jones, L., Sandberg, R. D. \& Sandham, N. D. 2010 Stability and Receptivity Characteristics of a Laminar Separation Bubble on an Aerofoil. Journal of Fluid Mechanics 648, 257-296.

Kays, W. \& Crawford, M. 1980 Convective Heat and Mass Transfer, 2nd edn. The address: McGraw-Hill Publishing Company.

Kennedy, C., Carpenter, M. \& Lewis, R. 2000 Low-storage, Explicit Runge-Kutta Schemes for the Compressible Navier-Stokes Equations. Applied Numerical Mathematics 35 (3).

Kennedy, C. \& Gruber, A. 2008 Reduced Aliasing Formulations of the Convective Terms within the Navier-Stokes Equations for a Compressible Fluid. Journal of Computational Physics 227 (3).

Moore, J. \& Elward, K. M. 1993 Shock Formation in Overexpanded Tip Leakage Flow. Journal of Turbomachinery $\mathbf{1 1 5}$.

Moore, J., Moore, J. G., Henry, G. S. \& Chaudhry, U. 1989 Flow and Heat Transfer in Turbine Tip Gaps. Journal of Turbomachinery 111. 
Sandberg, R., Pichler, R., Chen, L., Johnstone, R. \& Michelassi, V. 2015 Compressible Direct Numerical Simulation of Low-Pressure Turbines: Part I - Methodology. Journal of Turbomachinery 137, 051011-1-051011-10.

SANDBERG, R. D. 2012 Numerical investigation of turbulent supersonic axisymmetric wakes. Journal of Fluid Mechanics 702, 488-520.

SAndBerg, R. D. \& FAsel, H. F. 2006 Numerical Investigation of Transitional Supersonic Axisymmetric Wakes. Journal of Fluid Mechanics 563, 1-41.

Sandberg, R. D., Sandham, N. \& Suponitsky, V. 2012 DNS of Compressible Pipe Flow Exiting into a Coflow. International Journal of Heat and Fluid Flow 35, 33 - 44.

SandberG, R. D. \& Sandham, N. D. 2006 Nonreflecting Zonal Characteristic Boundary Condition for Direct Numerical Simulation of Aerodynamic Sound. AIAA Journal 44 (2).

Spalart, P. \& Coleman, G. 1997 Numerical Study of a Separation Bubble with Heat Transfer. European Journal of Mechanics B 16, 169-189.

Spalart, P. \& Strelets, M. 2000 Mechanisms of Transition and Heat Transfer in a Separation Bubble. Journal of Fluid Mechanics 403, 329-349.

Wheeler, A. \& Sandberg, R. D. 2012 Direct Numerical Simulations of a Transonic Turbine Tip Flow 13th International Symposium on Unsteady Aerodynamics, Aeroacoustics and Aeroelasticity of Turbomachines, University of Tokyo, Japan.

Wheeler, A. P. S., Atkins, N. R. \& He, L. 2011 Turbine Blade Tip Heat Transfer in Low Speed and High Speed Flows. Journal of Turbomachinery 133 (4).

Wheeler, A. P. S., Sandberg, R. D., Sandham, N. D., Pichler, R., Michelassi, V. \& Laskowski, G. 2016 Direct Numerical Simulations of a High-Pressure Turbine Vane. Journal of Turbomachinery 138.

Zhang, Q. \& He, L. 2013 Tip-Shaping for HP Turbine Blade Aerothermal Performance Management. Journal of Turbomachinery 135, 051025.

Zhang, Q., He, L. \& Rawlinson, A. 2014 Effects of Inlet Turbulence and End-Wall Boundary Layer on Aerothermal Performance of a Transonic Turbine Blade Tip. Journal of Engineering for Gas Turbines and Power 136, 052603.

Zhang, Q., O’Dowd, D. O., He, L., Wheeler, A. P. S., Ligrani, P. M. \& Cheong, B. C. Y. 2011 Overtip Shock Wave Structure and Its Impact on Turbine Blade Tip Heat Transfer. Journal of Turbomachinery 133 (4). 